\title{
NerveCheck for the Detection of Sensory Loss and Neuropathic Pain in Diabetes
}

\author{
Georgios Ponirakis, MPhil, ${ }^{1,2}$ Maria N. Odriozola, BSc, Samantha Odriozola, BSc, \\ loannis N. Petropoulos, PhD, ${ }^{1,2}$ Shazli Azmi, MBChB, Maryam Ferdousi, PhD, Hassan Fadavi, MD, \\ Uazman Alam, MBChB, Andrew Marshall, MBChB, Maria Jeziorska, MD, Anthony Miro, BSc, \\ Ahmad Kheyami, MD, Mitra Tavakoli, PhD, Ahmed Al-Ahmar, MD, Maria B. Odriozola, BSc, \\ Ariel Odriozola, MD, and Rayaz A. Malik, MBChB ${ }^{1,2}$
}

\begin{abstract}
Background: Accurate and economic detection of nerve damage in diabetes is key to more widespread diagnosis of patients with diabetic peripheral neuropathy (DPN) and painful diabetic neuropathy. This study examined the diagnostic performance of NerveCheck, an inexpensive (\$500) quantitative sensory testing (QST) device.

Methods: One hundred forty-four subjects (74 with and 70 without diabetes) underwent assessment with NerveCheck, neuropathy disability score (NDS), nerve conduction studies (NCS), intraepidermal and corneal nerve fiber density (IENFD and CNFD), and McGill questionnaire for neuropathic pain.

Results: Of the 74 subjects with diabetes, 41 were diagnosed with DPN based on the NDS. The NerveCheck scores for vibration perception threshold (VPT), cold perception threshold (CPT), and warm perception threshold (WPT) were significantly lower $(P \leq 0.0001)$ in diabetic patients with DPN compared to patients without DPN. The diagnostic accuracy of VPT was high with reference to NCS (area under the curve [AUC]: 82\%-84\%) and moderate for IENFD, CNFD, and neuropathic pain (AUC: 60\%-76\%). The diagnostic accuracy of CPT and WPT was moderate with reference to NCS, IENFD, and CNFD (AUC: 69\%-78\%) and low for neuropathic pain (AUC: $63 \%-65 \%$ ).

Conclusions: NerveCheck is a low-cost QST device with good diagnostic utility for identifying sensory deficits, comparable to established tests of large and small fiber neuropathy and for the severity of neuropathic pain.
\end{abstract}

Keywords: NerveCheck, Quantitative sensory testing, Diabetic peripheral neuropathy, Diagnostic device.

Introduction

$\mathbf{T}$ HE PREVALENCE OF diabetic peripheral neuropathy (DPN) has been reported to be up to $50 \%{ }^{1,2}$ About half of the people with DPN are affected by asymptomatic neuropathy, ${ }^{3}$ and therefore, an absence of symptoms does not mean an absence of DPN. Indeed neuropathy is central to the development of painful neuropathy and foot ulceration in patients with diabetes. ${ }^{4,5}$ Hence, it is important to identify early sensory deficits in patients with diabetes to improve the risk factors and limit the development and progression of DPN. Both sensory deficits and altered thresholds for sensory stimuli can be measured reliably using quantitative sensory testing (QST). ${ }^{6}$ A loss of vibration sensation is related to large fiber neuropathy, ${ }^{7}$ while loss of thermal sensation is related to small fiber neuropathy. Indeed, QST assessment has been endorsed for the quantification of sensory deficits by the NeuPSIG consensus, ${ }^{7}$ and the diagnosis of neuropathic

\footnotetext{
${ }^{1}$ Research Division, Weill Cornell Medicine-Qatar, Qatar Foundation, Education City, Doha, Qatar.

${ }^{2}$ Institute of Cardiovascular Sciences, Faculty of Biology, Medicine and Health, University of Manchester and Central Manchester NHS Foundation Trust, Manchester Academic Health Science Centre, Manchester, United Kingdom.

${ }^{3}$ Department of Electronics Engineering, Phi Med Europe Barcelona, Barcelona, Spain.

${ }^{4}$ ICEN, Catalonian Institute of Endocrinology and Nutrition, Medical Centre, Barcelona, Spain

(C) Georgios Ponirakis, et al., 2016; Published by Mary Ann Liebert, Inc. This Open Access article is distributed under the terms of the Creative Commons Attribution Noncommercial License (http://creativecommons.org/licenses/by-nc/4.0/) which permits any noncommercial use, distribution, and reproduction in any medium, provided the original author(s) and the source are credited.
} 
pain relies on a combination of positive neuropathic symptoms and sensory deficits as well as evoked pain. ${ }^{8}$ However, QST is subjective and the devices used to undertake the assessment are relatively expensive $(\$ 15,000)$ and therefore not widely used in daily clinical practice.

NerveCheck is an inexpensive (\$500), portable QST device that measures the vibration perception threshold (VPT), cold perception threshold (CPT), warm perception threshold (WPT), and the heat pain threshold (HPT) of the patient. It uses the method of levels where a series of predefined stimuli are applied over a broad range of intensities to produce a categorical output and define the severity of neuropathy. We have recently shown that it has good reproducibility and comparable diagnostic accuracy to established QST equipment for the diagnosis of DPN. ${ }^{9}$ It can also accurately identify sensory deficits to help in the identification of patients with painful diabetic neuropathy. ${ }^{10}$

Objective tests of small fiber neuropathy include structural loss of small nerve fibers detected by corneal confocal microscopy (CCM) in the eye and intraepidermal nerve fiber density (IENFD) in skin biopsies taken from the foot. ${ }^{11-13}$

In the present study, we have assessed the diagnostic utility of NerveCheck for detecting both large and small fiber neuropathy by comparing its diagnostic performance against nerve conduction studies (NCS), CCM, IENFD, and severity of painful diabetic neuropathy.

\section{Research Design and Methods}

Participants in the study were recruited from the Manchester Diabetes Centre, Manchester Royal Infirmary in Manchester, United Kingdom. The study was performed at the NIHR Wellcome Trust Clinical Research Facility between January 7, 2013 and September 19, 2014. Exclusion criteria included subjects with communication disorders, cognitive deficits, severe anxiety, severe depression, or history of neuropathy due to a nondiabetic cause. Control subjects with diabetes or suffering from any acute or chronic pain condition were excluded. All subjects were without any pain medication for at least $24 \mathrm{~h}$ before the investigation. This study was approved by the Local Research Ethics committee, and all patients gave informed consent to take part in the study. The research adhered to the tenets of the declaration of Helsinki.

\section{Demographic measures}

All study participants underwent assessment of glycated hemoglobin (HbA1c), body mass index (BMI), blood pressure, and cholesterol.

\section{Quantitative sensory testing}

Subjects were familiarized with the procedure and allowed to acclimatize for $10 \mathrm{~min}$ in the examination room. ${ }^{9}$ NerveCheck (Phi Med Europe S.L., Barcelona, Spain) applies the method of levels for a series of predefined stimuli (vibration intensity and heat waveform), and the method of limits for ramped stimuli $\left(1^{\circ} \mathrm{C} / \mathrm{s}\right)$ to the skin and, for each stimulus, the subject has to report when the stimulus is perceived to establish the VPT, CPT, and WPT or whether it is painful or not for the HPT. The output is categorical in terms of the degree of abnormality. The normal and abnormal range for VPT is $12-8$ and 7-0 and for CPT and WPT is 6-3 and 2-0. More information about the NerveCheck can be found online (www.phimedeurope.com).

\section{Nerve conduction studies}

Electrodiagnostic studies were undertaken using a Dantec "Keypoint"' system (Dantec Dynamics Ltd., Bristol, United Kingdom) equipped with a DISA temperature regulator to keep lower limb temperature constantly between $32^{\circ} \mathrm{C}$ and $35^{\circ} \mathrm{C}$. Sural nerve conduction velocity (SNCV), sural nerve action potential (SNAP), and peroneal motor nerve conduction velocity (PMNCV) were assessed in the right lower limb by a consultant neurophysiologist.

\section{Corneal confocal microscopy}

Patients underwent examination with the Heidelberg Retina Tomograph (HRT III RCM) in vivo corneal confocal microscope (Heidelberg Engineering $\mathrm{GmbH}$, Heidelberg, Germany) using our established methodology. ${ }^{14}$ Corneal nerve fiber density (CNFD), the total number of nerve fibers $\left(\mathrm{No} . / \mathrm{mm}^{2}\right)$, corneal nerve branch density, the total number of nerve branches (No./mm ${ }^{2}$ ), corneal nerve fiber length, and the total length of all nerve fibers and branches $\left(\mathrm{mm} / \mathrm{mm}^{2}\right)$ captured within the area of cornea were quantified from $\sim 5$ adjacent images/subject using the ACCMetrics, an automated image analysis software. ${ }^{14}$ ACCMetrics is available to all potential collaborators solely for research purposes (notfor-profit/noncommercial) and is protected by the University of Manchester in the form of a license agreement, which can be requested online (www.human-development.manchester .ac.uk/ena/ACCMetricsuserinstructions\#Researchlicence agreement).

\section{Intraepidermal nerve fiber density}

A $3 \mathrm{~mm}$ punch skin biopsy was taken from the dorsum of the foot under $1 \%$ lidocaine local anesthesia. Skin samples were immediately fixed in $4 \%$ (wt./vol.) paraformaldehyde for $24 \mathrm{~h}$ and then cryoprotected in sucrose for $18 \mathrm{~h}$ and cut into $50 \mu \mathrm{m}$ sections. Immunohistochemistry was performed as previously described. ${ }^{15}$ An image analysis camera AxioCam MRc (Ziess, Germany) and Leica QWin Standard V2.4 (Leica Microsystems Imaging, Cambridge, United Kingdom) were used to quantify IENFD, which is the total number of nerve fibers per millimeter length of epidermis (No./mm).

\section{Measurement of neuropathic pain}

The McGill Pain Questionnaire was utilized to assess the quality of the pain using descriptors such as throbbing, shooting, distressing, and excruciating. ${ }^{16}$ It contains a total of 78 pain descriptors, which can assess sensory and affective pain qualities.

Study definition of DPN. DPN was defined according to the neuropathy disability score (NDS). The maximum score for the NDS is 10, indicating a complete loss of all sensory responses and absent reflexes. Scores $>2$ are abnormal. ${ }^{5,17}$

\section{Statistical analysis}

We performed an unpaired $t$-test to assess quantitative variables between groups using GraphPad Prism, version 6.05. All data are expressed as mean \pm standard error of mean. A $P$-value $<0.05$ was considered statistically significant. 
Table 1. Comparison of Clinical Data of Subjects with Diabetes With and Without Diabetic Peripheral Neuropathy Defined by the Neuropathy Disability Score and Control Subjects

\begin{tabular}{|c|c|c|c|}
\hline & Control subjects & No neuropathy & Neuropathy \\
\hline$n$ & 70 & 41 & 33 \\
\hline Age & $41.8 \pm 1.63 * * *$ & $44.3 \pm 2.19$ & $64.1 \pm 1.79 * * *$ \\
\hline Duration of diabetes & - & $23.3 \pm 2.03$ & $37.6 \pm 3.2 * * *$ \\
\hline BP-sys (mm Hg) & $122.8 \pm 2.87 * * *$ & $129.9 \pm 3.1$ & $147.9 \pm 4.78 * *$ \\
\hline BMI $\left(\mathrm{kg} / \mathrm{m}^{2}\right)$ & $25.8 \pm 0.79 *$ & $27.6 \pm 0.87$ & $28.4 \pm 0.8$ \\
\hline HbA1c (\%) & $5.29 \pm 0.12 * * *$ & $7.5 \pm 0.18 * * *$ & $7.9 \pm 0.26$ \\
\hline HbA1c (mmol/mol) & $34.3 \pm 1.35 * * *$ & $59.1 \pm 1.97 * * *$ & $62.6 \pm 2.82 * * *$ \\
\hline NerveCheck VPT & $8.6 \pm 0.37 * * *$ & $6.4 \pm 0.75 * *$ & $2.1 \pm 0.6^{* * *}$ \\
\hline NerveCheck CPT & $5.9 \pm 0.07 * * *$ & $5.5 \pm 0.22 *$ & $3.2 \pm 0.46^{* * * *}$ \\
\hline NerveCheck WPT & $4.7 \pm 0.25^{* * *}$ & $5.7 \pm 0.15^{* *}$ & $2.5 \pm 0.4 * * *$ \\
\hline PMNCV (m/s) & $49.6 \pm 0.66 * * *$ & $42.9 \pm 0.88 * * *$ & $36.6 \pm 1.6^{* *}$ \\
\hline $\operatorname{SNCV}(\mu \mathrm{V})$ & $50.9 \pm 0.77 * * *$ & $44.2 \pm 0.92 * * *$ & $35.9 \pm 1.4 * * *$ \\
\hline SNAP (m/s) & $20.5 \pm 1.7 * * *$ & $12.3 \pm 1.02 * * *$ & $5.1 \pm 1.12 * * *$ \\
\hline CNFD (No. $/ \mathrm{mm}^{2}$ ) & $28.8 \pm 0.92 * * *$ & $22.9 \pm 1.3 * *$ & $14.2 \pm 1.6^{* * *}$ \\
\hline IENFD (No./mm) & $10.9 \pm 0.8 * * *$ & $8.05 \pm 0.7^{*}$ & $3.8 \pm 0.75 * *$ \\
\hline
\end{tabular}

Data are mean \pm standard error of the mean, $P$-values are derived from unpaired $t$-test: $* P \leq 0.05, * * P \leq 0.001, * * * P \leq 0.0001$, The $P$-values for comparing those with versus without DPN are in the left column, no DPN versus controls in the middle column, and DPN versus controls in the right column.

BMI, body mass index; CNFD, corneal nerve fiber density; CPT, cold perception threshold; DPN, diabetic peripheral neuropathy; HbA1c, glycated hemoglobin; IENFD, intraepidermal nerve fiber density; PMNCV, peroneal motor nerve conduction velocity; SNAP, sural nerve action potential; SNCV, sural nerve conduction velocity; VPT, vibration perception threshold; WPT, warm perception threshold.

Receiver operating characteristic (ROC) curve analysis was used to compare the diagnostic performance of NerveCheck against objective endpoints for large and small fiber neuropathy using GraphPad Prism, version 6.05. ROC curve analysis used the area under the curve (AUC) to determine the optimal sensitivity and specificity of the NerveCheck test.

\section{Results}

\section{Clinical data}

Of the 74 patients with diabetes, 33 were diagnosed with and 41 without DPN based on NDS and compared with 70 control subjects. The demographic and clinical characteristics of the subjects with and without DPN and controls are presented in Table 1. BMI and HbA1c did not differ, but age $(P \leq 0.0001)$, duration of diabetes $(P \leq 0.0001)$, and systolic blood pressure $(P \leq 0.001)$ were significantly greater in those with compared to those without DPN. Using NerveCheck, the group with DPN had significantly lower scores for VPT $(P \leq 0.0001)$, CPT $(P \leq 0.0001)$, and WPT $(P \leq 0.0001)$. With NerveCheck, 44 out of 74 patients with diabetes were detected to have an abnormal vibration or thermal threshold.
The group with DPN also had a significantly lower PMNCV, sural nerve action potential (SNAP) and conduction velocity (SNCV) ( $P \leq 0.0001$ for all comparisons), CNFD $(P \leq 0.0001)$, and IENFD $(P \leq 0.001)$.

\section{Detection of sensory deficits in asymptomatic and painful neuropathy}

The incidence of sensory loss detected by NerveCheck in the presence and absence of numbness and neuropathic pain is shown in Table 2. Subjects with diabetes had significantly higher sensory deficits than the control group $(P \leq 0.05-$ $0.0001)$. The percentage of sensory deficit in subjects without numbness was $43 \%(12 / 48)$, whereas in subject with numbness it was $65 \%$ (30/45). An abnormal vibration or thermal response was significantly more likely to occur in subjects with numbness ( $P \leq 0.0001$ for all comparisons).

The incidence of sensory deficit in subjects without painful symptoms was $50 \%(28 / 56)$, whereas in subjects with painful symptoms it was $78 \%$ (14/18). Sensory deficits were significantly more likely to occur in subjects with pain compared to those without pain (VPT and WPT, $P \leq 0.05$ ).

Table 2. Comparison of the NerveCheck Results of Controls, Subjects with Diabetes With and Without Numbness and With and Without Neuropathic Pain Defined by the McGill Pain Questionnaire

\begin{tabular}{lccccc}
\hline Deficits & Controls & No numbness & Numbness & No pain & Pain \\
\hline$n$ & 69 & 28 & 45 & 18 & 56 \\
Signs/Total No. of subjects (\%) & $4 / 69(6)$ & $12 / 28(43)$ & $30 / 45(67)$ & $28 / 56(50)$ & $14 / 18(78)$ \\
NerveCheck VPT & $8.98 \pm 0.37 * * *, * * *$ & $5.4 \pm 1.02$ & $3.75 \pm 0.68 * * *$ & $5 \pm 0.65 * *$ & $2.67 \pm 0.9 *$ \\
NerveCheck CPT & $5.89 \pm 0.07 * * *, * * *$ & $5.3 \pm 0.28$ & $4.11 \pm 0.38 * * *$ & $4.78 \pm 0.28 * * *$ & $3.61 \pm 0.66$ \\
NerveCheck WPT & $4.74 \pm 0.25 * * * *$ & $5.15 \pm 0.31 * *$ & $3.88 \pm 0.4 * * *$ & $4.72 \pm 0.29$ & $3.17 \pm 0.63 *$ \\
\hline
\end{tabular}

Data are mean \pm standard error of the mean, $P$-values are derived by unpaired $t$-test: $* P \leq 0.05, * * P \leq 0.001, * * * P \leq 0.0001$. The $P$-values for controls versus numbness and painful symptoms are in the control column, respectively, controls versus no symptoms are in the no symptom columns, and present versus absent symptoms are in the symptom columns. 
NerveCheck diagnostic performance against

large and small fiber neuropathy

and neuropathic painful symptoms

The diagnostic performance of NerveCheck was compared to large fiber dysfunction, small fiber loss, and severity of neuropathic pain symptoms using NCS, CCM, IENFD, and McGill questionnaire, respectively, as reference methods (Fig. 1). The diagnostic performance expressed in AUC\% using ROC analysis is shown in Table 3. Vibration perception using NerveCheck had an AUC of $82 \%$ (95\% confidence interval [CI] 72-93), $P<0.0001$ for SNAP, and $84 \%$ (95\% CI 75-94), $P<0.0001$ for SNCV. The sensitivity and specificity of VPT against SNCV were $88 \%$ (95\% CI 67-97) and 82\% (95\% CI 71-90), respectively. The AUC of the cold perception testing was $78 \%$ (95\% CI 66-91), $P<0.0001$ for CNFD, and 70\% (95\% CI $54-87), P=0.01$ for IENFD. The sensitivity and specificity of CPT against CNFD were 67\% (95\% CI 44-84) and 85\% (95\% CI 75-92), respectively, and against IENFD were $53 \%$ (95\% CI 28-77) and 82\% (95\% CI 67-91), respectively. The AUC of WPT was 71\% (95\% CI 59-84), $P=0.002$ for CNFD, and $69 \%$ (95\% CI 52-85), $P=0.03$ for IENFD. The sensitivity and specificity of WPT against CNFD were 67\% (95\% CI 44-84) and 76\% (95\% CI 64$85)$, respectively, and against IENFD were $56 \%$ (95\% CI 29-81) and $81 \%$ (95\% CI 67-91), respectively. For the severity of neuropathic pain, VPT had an AUC of $70 \%$ (95\% CI 57-83), $P=0.006$, and a sensitivity and specificity of $70 \%$ (95\% CI 57-83) and 68\% (95\% CI 56-78), respectively. For CPT, the AUC was $63 \%$ (95\% CI 48-78), $P=0.07$, and the sensitivity and specificity were $40 \%(95 \%$ CI 19-64) and 84\% (95\% CI 75-91), respectively. For WPT, the AUC was $65 \%$ (95\% CI 50-80), $P=0.04$, and the sensitivity and specificity were $55 \%$ (95\% CI 32-77) and $73 \%$ (95\% CI 61-82), respectively.
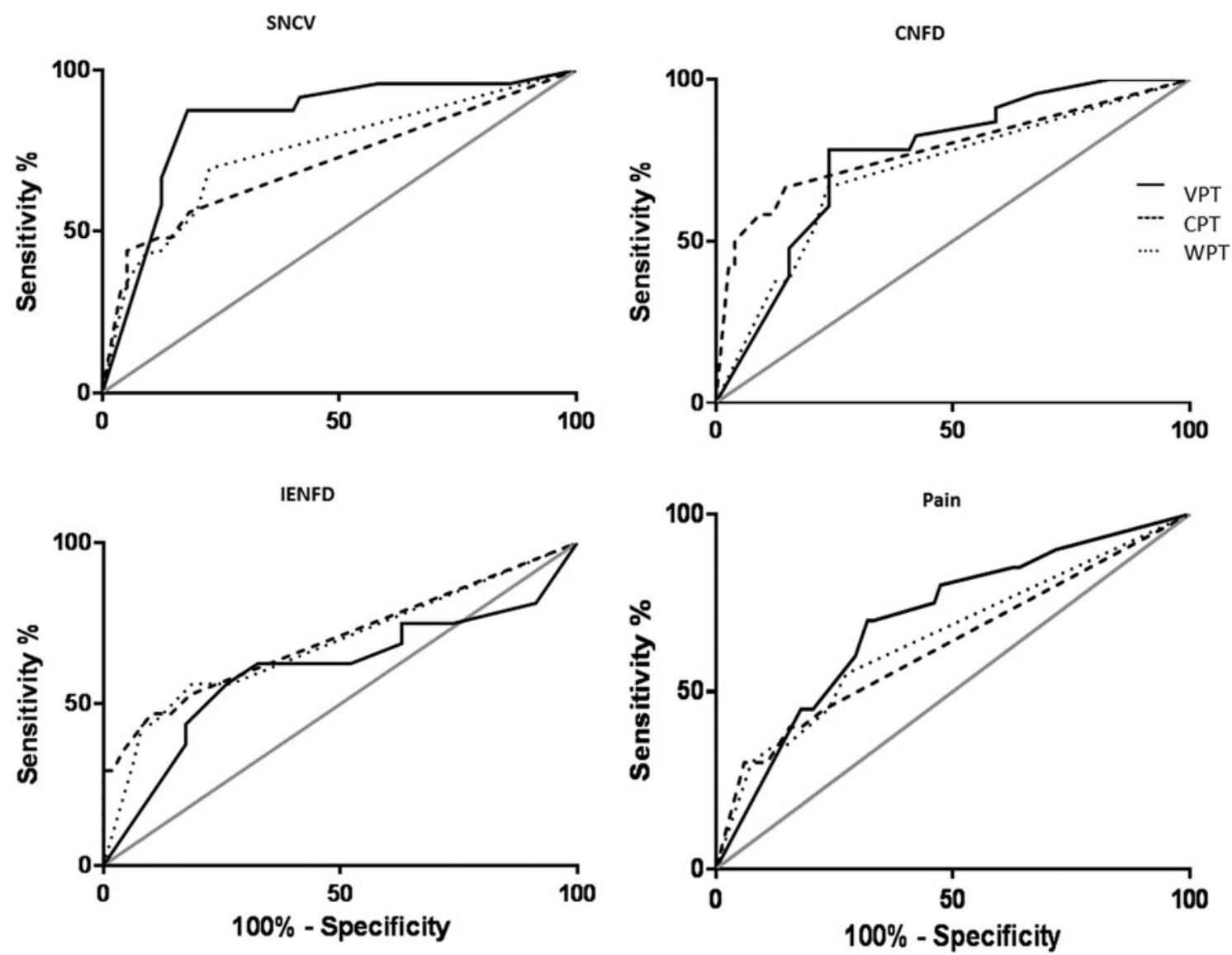

FIG. 1. ROC curve analysis was used to compare the diagnostic accuracy of the VPT (black line), CPT (dashed line), and WPT (dotted line) of the NerveCheck in detecting large nerve fiber damage using SNCV, small nerve fiber damage using CNFD, and IENFD and neuropathic pain using the McGill questionnaire. The gray line is the null value of the ROC curve. The AUC of the NerveCheck against the SNCV was 84\% (95\% CI 75-94) for the VPT, 71\% (95\% CI 58-84) for the CPT, and 75\% (95\% CI 63-87) for the WPT, against the CNFD was 76\% (95\% CI 66-87) for the VPT, 78\% (95\% CI 66-91) for the CPT, and 71\% (95\% CI 59-84) for the WPT, against the IENFD was 60\% (95\% CI 42-79) for the VPT, 70\% (95\% CI 54-87) for the CPT, and 69\% (95\% CI 52-85) for the WPT, and against neuropathic pain was 70\% (95\% CI 57-83) for the VPT, 63\% (95\% CI 48-78) for the CPT, and 65\% (95\% CI 50-80) for the WPT. AUC, area under the curve; CI, confidence interval; CNFD, corneal nerve fiber density; CPT, cold perception threshold; IENFD, intraepidermal nerve fiber density; ROC, receiver operating characteristic; SNCV, sural nerve conduction velocity; VPT, vibration perception threshold; WPT, warm perception threshold. 
Table 3. Receiver Operating Characteristic Curve Analysis Was Used to Evaluate the Diagnostic Performance of NerveCheck for Sensory loss Against Sural Nerve Action Potential,

Sural Nerve Conduction Velocity, Corneal Nerve Fiber Density (No./Mm²), Intraepidermal Nerve Fiber Density (No./mm), and McGill Questionnaire for Symptoms of Painful Neuropathy

\begin{tabular}{lccc}
\hline & $V P T, A U C \%(95 \% C I), \mathrm{P}$ & $C P T, A U C \%(95 \% C I), \mathrm{P}$ & $W P T, A U C \%(95 \% C I), \mathrm{P}$ \\
\hline SNAP & $82(72-93),<0.0001$ & $70(56-84), 0.005$ & $78(65-90), 0.0002$ \\
SNCV & $84(75-94),<0.0001$ & $71(58-84), 0.002$ & $75(63-87), 0.0003$ \\
CNFD & $76(66-87), 0.0002$ & $78(66-91),<0.0001$ & $71(59-84), 0.002$ \\
IENFD & $60(42-79), 0.2$ & $70(54-87), 0.01$ & $69(52-85), 0.03$ \\
McGill (pain) & $70(57-83), 0.006$ & $63(48-78), 0.07$ & $65(50-80), 0.04$ \\
\hline
\end{tabular}

AUC, area under the curve; CI, confidence interval.

\section{Discussion}

In the present study, we have tested the clinical utility of NerveCheck, an inexpensive $(\$ 500)$ portable $(9.5 \times 6.1 \times$ $23.6 \mathrm{~cm}$ ) device that performs vibration, thermal, and HPT testing. NerveCheck identifies significantly more sensory deficits in people with either numbness in the feet or neuropathic pain compared to those without neuropathic symptoms. This study has specifically established the diagnostic accuracy of vibration and thermal testing using NerveCheck, against other established methods for the diagnosis of large and small fiber neuropathy. VPT testing optimally identifies large fiber dysfunction, and CPT testing optimally identifies a reduction in CNFD and IENFD.

DPN is defined by the presence of symptoms and/or signs of neuropathy. The diagnosis of DPN is confirmed with a history of neuropathic symptoms and a careful assessment of neurological deficits. Identifying early neuropathy is key to increased awareness, which might prevent the long-term sequelae of foot ulceration and amputation as well as helping to identify those with neuropathic as opposed to nociceptive pain. Neurological examination and monofilament testing identify advanced rather than early neuropathy, especially small fiber damage. ${ }^{17}$ This study shows that NerveCheck is an inexpensive device, which can easily perform QST to identify patients with sensory deficits.

Patients with diabetes for more than 10 years and loss of sensation in the foot are at high risk of developing foot ulcers. ${ }^{18}$ In a study of 6487 patients with diabetes, the prevalence of large fiber neuropathy defined by VPT $(\geq 15 \mathrm{~V})$ was reported to be $21 \%(19 \%-22 \%)$ in patients with diabetes duration $<5$ years and $37 \%(35 \%-39 \%)$ in patients with diabetes duration $>10$ years. ${ }^{2}$ In the United Kingdom Prospective Diabetes Study (UKPDS), the prevalence of severe large fiber neuropathy defined by VPT $(>25 \mathrm{~V})$ was reported to be $12 \%$ in patients with newly diagnosed type 2 diabetes. ${ }^{19}$ We have shown that VPT assessed using NerveCheck has high sensitivity and specificity compared to the Neurothesiometer, an established method for VPT testing. ${ }^{9}$ NerveCheck provides a simple categorical output, which can be easily interpreted in relation to the severity of neuropathy and hence allows risk stratification.

The diagnosis of painful diabetic peripheral neuropathy (PDPN) is based on a combination of painful neuropathic symptoms and eliciting sensory deficits and evoked pain ${ }^{8}$ and occurs in $20 \%-30 \%$ of patients with diabetes. ${ }^{20,21}$ Painful symptoms include burning, tingling, electric shock, or stabbing sensations, which are generally worse at night and dis- turb sleep. We have shown that the prevalence of painful symptoms assessed using the neuropathy symptom score (NSS) (>6) and PDPN assessed using the NSS (>6) and NDS $(>2)$ was $34 \%$ and $21 \%$, respectively, in 15,692 communitybased patients with diabetes from Northwest England. ${ }^{21}$ Furthermore, PDPN is significantly more likely to occur in patients with more severe DPN. ${ }^{22-24}$ Indeed, we also show that NerveCheck is significantly more likely to be abnormal in subjects with painful symptoms compared to those without pain.

Since QST detects both large and small fiber neuropathy, its diagnostic performance has been compared to established measures of large and small fiber neuropathy using NCS and IENFD, respectively. A study comparing QST of mechanical testing and NCS reported $75 \%$ sensitivity and $89 \%$ specificity for the detection of neuropathy. ${ }^{25}$ In the present study, the sensitivity and specificity of VPT using NerveCheck against SNCV were $88 \%$ and $82 \%$, respectively. The sensitivity of thermal testing compared to IENFD ranges from $36 \%$ to $100 \% .^{26}$ Our data show that the sensitivity and specificity of the CPT using NerveCheck were $53 \%$ and $82 \%$ for IENFD, and $67 \%$ and $85 \%$ for CNFD, respectively, indicating earlier structural compared to functional deficits.

In conclusion, the current study shows that NerveCheck, an inexpensive portable QST device, can identify abnormal vibration and thermal perception thresholds with good diagnostic utility compared to established tests of large and small fiber neuropathy and for the severity of neuropathic pain.

\section{Acknowledgments}

The NerveCheck device was provided by Dr. Ariel Odriozola, Phi Med Europe S.L. Barcelona, Spain. The authors thank the staff at NIHR/Wellcome Trust Clinical Research Facility in Central Manchester University Hospitals NHS Foundation Trust and its Deputy Director, Mr. Paul Brown, for providing a high-quality service and their stateof-the-art facility to carry out the research. Special thank you to the Nurse Manager, Mr. Ciaran Kilkelly, and the study Lead Nurse, Mr. Stephen Mawn and Mr. Kamlesh Patel, who offered a very professional service to our study subjects and for always being extra helpful. This study was funded by Phi Med Europe S.L. Barcelona, Spain, the National Institutes of Health (NIH) Grant 5RO1 NS46259-03 NINDS, and the Juvenile Diabetes Research Foundation (JDRF) Grant 52002-185. 


\section{Author Disclosure Statement}

G.P. researched data and wrote the article. A.O., M.N.O., S.O., A.M., and M.B.O. designed and created the NerveCheck. I.N.P., S.A., M.F., H.F., U.A., A.M., A.K., M.T., A.A.-A., and M.J. researched data. R.A.M. reviewed and edited the article and is the guarantor of this work. Phi Med Europe S.L. provided the NerveCheck device and funded half of the study. M.N.O., S.O., M.B.O., and A.O. are the owners and inventors of the NerveCheck. The authors confirm that the article has been read and approved by all named authors and that there are no other persons who satisfied the criteria for authorship and are not listed. They further confirm that the order of authors listed in the article has been approved by all of them.

\section{References}

1. Tesfaye $S$ : Recent advances in the management of diabetic distal symmetrical polyneuropathy. J Diabetes Investig 2011; 2:33-42.

2. Young MJ, Boulton AJ, MacLeod AF, et al.: A multicentre study of the prevalence of diabetic peripheral neuropathy in the United Kingdom hospital clinic population. Diabetologia 1993;36:150-154.

3. Boulton AJ, Vinik AI, Arezzo JC, et al.: Diabetic neuropathies: a statement by the American Diabetes Association. Diabetes Care 2005;28:956-962.

4. Malik R, Veves A, Tesfaye S, et al.: Small fiber neuropathy: role in the diagnosis of diabetic sensorimotor polyneuropathy. Diabetes Metab Res Rev 2011;27:678-684.

5. Tesfaye S, Boulton AJ, Dyck PJ, et al.: Diabetic neuropathies: update on definitions, diagnostic criteria, estimation of severity, and treatments. Diabetes Care 2010;33:22852293.

6. Gruener G, Dyck PJ: Quantitative sensory testing: methodology, applications, and future directions. J Clin Neurophysiol 1994;11:568-583.

7. Backonja MM, Attal N, Baron R, et al.: Value of quantitative sensory testing in neurological and pain disorders: NeuPSIG consensus. Pain 2013;154:1807-1819.

8. Treede RD, Jensen TS, Campbell JN, et al.: Neuropathic pain: redefinition and a grading system for clinical and research purposes. Neurology 2008;70:1630-1635.

9. Ponirakis G, Odriozola MN, Odriozola S, et al.: NerveCheck: an inexpensive quantitative sensory testing device for patients with diabetic neuropathy. Diabetes Res Clin Pract 2016;113:101-107.

10. Haanpaa M, Attal N, Backonja M, et al.: NeuPSIG guidelines on neuropathic pain assessment. Pain 2011;152:14-27.

11. Hafner J, Lee G, Joester J, et al.: Thermal quantitative sensory testing: a study of 101 control subjects. J Clin Neurosci 2015;22:588-591.

12. Petropoulos IN, Green P, Chan AW, et al.: Corneal confocal microscopy detects neuropathy in patients with type 1 diabetes without retinopathy or microalbuminuria. PLoS One 2015;10:e0123517.

13. Chen X, Graham J, Dabbah MA, et al.: Small nerve fiber quantification in the diagnosis of diabetic sensorimotor polyneuropathy: comparing corneal confocal microscopy with intraepidermal nerve fiber density. Diabetes Care 2015; 38:1138-1144.

14. Dabbah MA, Graham J, Petropoulos IN, et al.: Automatic analysis of diabetic peripheral neuropathy using multi-scale quantitative morphology of nerve fibres in corneal confocal microscopy imaging. Med Image Anal 2011;15:738-747.

15. Quattrini C, Jeziorska M, Tavakoli M, et al.: The Neuropad test: a visual indicator test for human diabetic neuropathy. Diabetologia 2008;51:1046-1050.

16. Melzack R: The McGill Pain Questionnaire: major properties and scoring methods. Pain 1975;1:277-299.

17. Abbott CA, Carrington AL, Ashe H, et al.: The North-West Diabetes Foot Care Study: incidence of, and risk factors for, new diabetic foot ulceration in a community-based patient cohort. Diabet Med 2002;19:377-384.

18. DiPreta JA: Outpatient assessment and management of the diabetic foot. Med Clin North Am 2014;98:353-373.

19. Genuth S, Eastman R, Kahn R, et al.: Implications of the United kingdom prospective diabetes study. Diabetes Care 2003;26(Suppl 1):S28-S32.

20. Davies M, Brophy S, Williams R, Taylor A: The prevalence, severity, and impact of painful diabetic peripheral neuropathy in type 2 diabetes. Diabetes Care 2006;29: 1518-1522.

21. Abbott CA, Malik RA, van Ross ER, et al.: Prevalence and characteristics of painful diabetic neuropathy in a large community-based diabetic population in the U.K. Diabetes Care 2011;34:2220-2224.

22. Sorensen L, Molyneaux L, Yue DK: Insensate versus painful diabetic neuropathy: the effects of height, gender, ethnicity and glycaemic control. Diabetes Res Clin Pract 2002;57:45-51.

23. Veves A, Manes C, Murray HJ, et al.: Painful neuropathy and foot ulceration in diabetic patients. Diabetes Care 1993; 16:1187-1189.

24. Themistocleous AC, Ramirez JD, Shillo PR, et al.: The Pain in Neuropathy Study (PiNS): a cross-sectional observational study determining the somatosensory phenotype of painful and painless diabetic neuropathy. Pain 2016;157: 1132-1145.

25. Blankenburg M, Kraemer N, Hirschfeld G, et al.: Childhood diabetic neuropathy: functional impairment and non-invasive screening assessment. Diabet Med 2012;29:1425-1432.

26. Lauria G, Cornblath DR, Johansson O, et al.: EFNS guidelines on the use of skin biopsy in the diagnosis of peripheral neuropathy. Eur J Neurol 2005;12:747-758.

Address correspondence to:

Rayaz A. Malik, MBChB

Research Division

Weill Cornell Medicine-Qatar

Qatar Foundation

Education City

PO Box: 24144

Doha

Qatar

E-mail: ram2045@qatar-med.cornell.edu 